\author{
Cadernos de \\ ESTUDOS LINGUÍSTIICOS - (59.3), Campinas, pp. 537-553 - set./dez. 2017
}

\title{
FATORES DETERMINANTES NA ASSOCIAÇÃO TONAL EM SENTENÇAS NEUTRAS DO PORTUGUÊS BRASILEIRO
}

\author{
FLAVIANE ROMANI FERNANDES-SVARTMAN ${ }^{1}$ \\ (USP) \\ NÍCOLAS ROMANO \\ (UNICAMP)
}

\begin{abstract}
RESUMO: Este trabalho tem como objetivo a investigação dos fatores determinantes na associação de acentos tonais ao contorno entoacional das sentenças declarativas neutras do português brasileiro (PB). Nossa hipótese é que, além de fatores como número de sílabas e ramificação sintática e prosódica, a posição sintática ocupada pelas palavras prosódicas (PW) nas sentenças declarativas neutras também pode desempenhar um papel relevante na associação de acentos tonais ao contorno entoacional dessas sentenças em PB. Para o alcance de nosso objetivo, foram analisados entoacionalmente dados de fala da variedade paulista do PB, à luz da visão integrada entre as teorias Fonologia Entoacional (PIERREHUMBERT, 1980; BECKMAN; PIERREHUMBERT, 1986; LADD 1996, 2008) e Fonologia Prosódica (SELKIRK, 1984, 1986 2000; NESPOR; VOGEL, 1986, 2007) e com o uso da ferramenta de análise de fala PRAAT (BOESRMA; WEENINK 2010). Os resultados obtidos confirmaram a hipótese deste trabalho, uma vez que revelaram que a associação de um acento tonal a cada PW que compõe os sujeitos é praticamente categórica e que é possível a associação de acento tonal apenas à PW cabeça de frase fonológica nos predicados das sentenças. Tal diferença entre a associação tonal nesses constituintes sintáticos resulta em uma densidade tonal média maior (comprovada estatisticamente) no sujeito do que nos predicados das sentenças de nosso corpus.
\end{abstract}

Palavras-chave: Português brasileiro; Entoação (Fonologia); Associação tonal.

ABSTRACT: This paper aims to investigate the determinant factors in the association of pitch accents with the intonational contour of declarative neutral sentences of Brazilian Portuguese (BP). Our hypothesis is that in addition to factors such as number of syllables and syntactic and prosodic branching, the syntactic position occupied by prosodic words (PW) in neutral declarative sentences may also play a relevant role in the association of pitch accents with the intonational contour of these sentences in BP. In order to reach our goal, we have analyzed intonationally speech data of the paulista variety of BP in the light of the integrated vision between the Intonational Phonology theory (PIERREHUMBERT, 1980; BECKMAN; PIERREHUMBERT, 1986; LADD 1996, 2008) and the Prosodic Phonology theory (SELKIRK, 1984, 1986; NESPOR; VOGEL, 1986, 2007) and with the use of the software PRAAT (BOESRMA; WEENINK 2010). The results obtained confirmed the hypothesis of this paper, since they revealed that the association of a pitch accent with each PW that composes the subjects is practically categorical and that it is possible the association of tonal pitch accents only with the PW head of phonological phrases in the predicates of sentences. The difference between the tonal association in these syntactic constituents results in a higher tonal density (statistically confirmed) in the subject than in the predicates of the sentences of our corpus.

Key-words: Brazilian Portuguese; Intonation (Phonology); Tonal association.

\footnotetext{
${ }^{1}$ USP - Universidade de São Paulo. São Paulo - São Paulo - Brasil / flavianesvartman@usp.br

${ }^{2}$ UNICAMP - Universidade Estadual de Campinas. Campinas - São Paulo - Brasil / nicolas. romano1995@gmail.com
} 


\section{INTRODUÇÃ ${ }^{3}$}

Na literatura fonológica sobre entoação em português brasileiro (doravante, PB), coerente com a visão integrada entre a Fonologia Autossegmental Métrica de análise etoacional (PIERREHUMBERT, 1980; BECKMAN; PIERREHUMBERT, 1986; LADD 1996, 2008)4 e a Fonologia Prosódica (SELKIRK, 1984, 1986, 2000; NESPOR; VOGEL, 1986, 2007), encontram- se divergências entre os pesquisadores quanto ao domínio prosódico relevante na atribuição de acentos tonais em sentenças neutras dessa variedade de português. ${ }^{5}$ Conforme Frota e Vigário (2000) e Tenani (2002), o domínio relevante para a associação de acentos tonais nas sentenças neutras do PB é a frase fonológica (PPh). ${ }^{6}$ Já Fernandes (2007) e Tenani e Fernandes-Svartman (2008) afirmam que, no mesmo tipo de sentença do PB, é a palavra prosódica (PW) o domínio relevante para a associação dos acentos tonais. ${ }^{7}$ Por sua vez, para Vigário e Fernandes-Svartman (2010), o domínio relevante para a mesma associação é o grupo de palavras prosódicas (PWG) proposto por Vigário $(2007,2010) .{ }^{8}$ Por fim, o trabalho de Toneli (2014) confirma, com base em análises estatísticas de dados, a afirmação de Fernandes (2007) e Tenani e Fernandes-Svartman (2008) de que o domínio relevante para a associação de acentos tonais em sentenças neutras do PB é, de fato, a palavra prosódica.

${ }^{3}$ Uma versão preliminar deste trabalho, desenvolvido no âmbito dos projetos "Fraseamento entoacional em português brasileiro" (processo FAPESP 2011/50044-9), "Interactive Atlas of the Prosody of Portuguese" (processo FCT PTDC/CLE-LIN/119787/2010) e "Fraseamento prosódico em português: comparações entre as variedades brasileira e africanas" (processo CNPq 459634/20143 3: do português arcaico e do português brasileiro contemporâneo" do II Congresso Internacional de Linguística Histórica, realizado de 07 a 10 de fevereiro de 2012, na Escola Politécnica da Universidade de São Paulo.

${ }^{4}$ Essa teoria é comumente chamada, na literatura brasileira sobre prosódia, de "Fonologia Entoacional" e este será o termo que utilizaremos neste artigo de agora em diante.

${ }^{5}$ Sobre acentos tonais, ver a subseção 2.1 .

${ }^{6} \mathrm{~A}$ frase fonológica ou sintagma fonológico ( $\mathrm{PPh}$ - phonological phrase) corresponde ao domínio de uma categoria sintática máxima (XP) nas teorias end-based e, nas teorias relationbased de Fonologia Prosódica, ao domínio que abrange, inicialmente, um núcleo lexical e todos os elementos funcionais do lado oposto à recursividade sintática até o próximo núcleo lexical. Sobre esse domínio em português, conferir Frota (2000), Sandalo e Truckenbrodt (2002), Tenani (2002), entre outros.

${ }^{7}$ Em linhas gerais, a palavra prosódica ( $\mathrm{PW}$ - prosodic word) pode ser definida como o domínio prosódico no qual pode haver apenas um acento primário (ou lexical). Conferir Schwindt (2000), Vigário (2003), Simioni (2008) e Toneli (2014) sobre esse domínio em português.

${ }^{8}$ Conforme a proposta de Vigário $(2007,2010)$, o grupo de palavras prosódicas (PWG - prosodic word group) é um constituinte localizado entre a palavra prosódica e a frase fonológica e que agrupa membros de diversos tipos de expressões compostas (como palavras compostas e siglas, por exemplo), mas que não desempenha papel relevante na organização prosódica dos clíticos, consistindo, portanto, em um domínio parcialmente diferente do grupo clítico (C - clitic group) (NESPOR; VOGEL, 1986; HAYES, 1989). 
Independentemente da discordância entre esses trabalhos quanto a essa temática, todos eles apresentam indícios de que fatores como número de sílabas antecedendo o acento principal da frase fonológica e ramificação sintática e prosódica dos constituintes são determinantes na associação de acentos tonais às sentenças neutras do PB.

$\mathrm{O}$ presente trabalho visa justamente à investigação dos fatores determinantes na associação de acentos tonais às sentenças neutras do PB, com base na análise de dados de fala da variedade paulista.

Levando em consideração o fato de que, em PB, a posição ocupada pelo sujeito das sentenças é um local de maior proeminência, dadas as particularidades sintáticas associadas a esse constituinte nessa variedade de português (sujeito-tópico por PB ser uma variedade orientada para o tópico, segundo PONTES, 1983; GALVES, 1998, 2001; entre outros), nossa hipótese é que, além de fatores como número de sílabas e ramificação sintática e prosódica, a posição sintática ocupada pelas PWs nas sentenças também pode desempenhar um papel relevante na associação de acentos tonais ao contorno entoacional neutro.

Este trabalho se encontra organizado nas próximas quatro seções. $\mathrm{Na}$ seção 2, apresentaremos o quadro teórico no qual se ancora a análise realizada. $\mathrm{Na}$ seção seguinte, descreveremos o corpus e a metodologia empregados no desenvolvimento do trabalho. Por sua vez, na seção 4, serão expostos os resultados obtidos, bem como a análise desses resultados. Finalmente, na quinta e última seção, apresentaremos nossas considerações finais.

\section{QUADRO TEÓRICO}

Os dados de fala são aqui analisados à luz das seguintes teorias fonológicas de análise prosódica: Fonologia Entoacional (PIERREHUMBERT, 1980; BECKMAN; PIERREHUMBERT, 1986; LADD, 1996, 2008; entre outros) e Fonologia Prosódica (SELKIRK, 1984, 1986, 2000; NESPOR; VOGEL, 1986, 2007; entre outros).

A adoção dessas duas teorias fonológicas de análise prosódica se justifica na medida em que assumimos uma visão integrada de entoação e organização dos domínios prosódicos (HAYES; LAHIRI, 1991; FROTA, 2000; TENANI, 2002; entre outros). Conforme tal visão, há correspondência entre os domínios delimitados entoacionalmente e os derivados de algoritmos da Fonologia Prosódica.

Uma evidência dessa visão é a possibilidade de correspondência entre domínios de regras rítmicas (e.g., retração de acentos), segmentais (e.g., sândi externo e haplologia sintática), de atribuição de tons e de alongamentos de fronteiras (FROTA, 2000). Em PB, por exemplo: (i) a regra de retração de acentos se dá no domínio da frase fonológica (ABOUSALH, 1997; SANDALO; TRUCKENBRODT, 2002), como ilustrado em (1); (ii) o sândi vocálico externo e a haplologia sintática não ocorrem entre duas palavras prosódicas quando a segunda é cabeça de frase fonológica, ou seja, porta o acento principal desse constituinte (conferir ABAURRE, 1994 e TENANI, 2002 para a afirmação sobre sândi vocálico externo e TENANI, 
2002 para a afirmação sobre haplologia sintática), como demonstrado no exemplo em (2) $;{ }^{9} \mathrm{e}$ (iii) a atribuição de acentos tonais é obrigatória à cabeça de frase fonológica (FROTA; VIGÁRIO, 2000; TENANI, 2002; FERNANDES, 2007; entre outros), como pode ser constatado em (3).

$$
\text { [café quente]PPh; *[café] } \mathrm{PPh}[\text { queima }] \mathrm{PPh}^{10}
$$

$$
*[\operatorname{lev}(\mathrm{o}) \text { uvas }] \mathrm{PPh} ;{ }^{*}[\mathrm{Matil}(\mathrm{de})] \mathrm{PPh}[\text { disse }] \mathrm{PPh}^{11}
$$

a. [(aluna)PWd (jovem)PWd $] \mathrm{PPh}^{12}$<smiles>C[In]C</smiles>

b. [(aluna)PWd (jovem)PWd]PPh

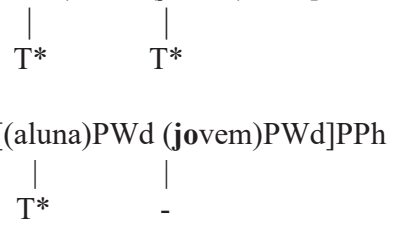

Outra evidência da visão integrada entre entoação e organização dos domínios prosódicos consiste no fato de a correspondência entre os domínios prosódicos estabelecidos entoacionalmente (JUN, 1996) e os derivados pela Fonologia Prosódica não se dar apenas a partir da frase entoacional (I), ${ }^{13}$ mas também a partir de domínios mais baixos. Por exemplo, em PB, a palavra prosódica e a frase fonológica são domínios relevantes na atribuição de acentos tonais (FROTA; VIGÁRIO, 2000; TENANI, 2002; FERNANDES, 2007; TONELI, 2104), uma vez que é frequente a atribuição de acento tonal a palavras prosódicas não cabeça de frase fonológica (ver exemplo em (3b)) e é

\footnotetext{
${ }^{9} \mathrm{O}$ sândi vocálico externo pode ser entendido como um processo resultante do encontro entre duas vogais adjacentes em fronteiras de palavras e que desencadeia reestruturações silábicas. Segundo Bisol (1996), o sândi vocálico externo compreende três processos: elisão (EL) - ex.: pala[vri]scrita "palavra escrita"; ditongação (DT) - ex.: casa[kwa]massado "casaco amassado"; e degeminação (DG) - ex.: ca[za] lugada "casa alugada". Por sua vez, tem-se a haplologia sintática quando se omite uma de duas sílabas iguais ou semelhantes contíguas em palavras prosódicas diferentes: caldo de cana > cal de cana.

${ }^{10}$ As sílabas sublinhadas representam sílabas portadoras de acento. O exemplo em (1) é adaptado de Sandalo e Truckenbrodt (2002).

${ }^{11}$ As sílabas em negrito representam sílabas que portam o acento principal de frase fonológica e os segmentos entre parêntenses representam elementos elididos por sândi vocálico externo, no primeiro exemplo, e por haplologia sintática, no segundo exemplo.

${ }^{12}$ As sílabas em negrito representam sílabas que portam o acento principal de frase fonológica; "T*" representa acento tonal; e "-" representa ausência de acento tonal.

${ }^{13} \mathrm{O}$ domínio da frase entoacional ou sintagma entoacional (I - intonational phrase) em português consiste em, conforme Frota (2000, pp. 57): “(i) all the $\phi s$ in a string that is not structurally attached to the sentence tree (i.e. parenthetical expression, tag questions, vocatives, etc);(ii) any remaining sequence of adjacent $\phi$ s in a root sentence; (iii) the domain of an intonation contour, whose boundaries coincide with the positions in which grammar-related pauses may be introduced in an utterance". Para maiores detalhes sobre esse constituinte prosódico em português, conferir, entre outros: Frota (2000), Tenani (2002) e Serra (2009).
} 
obrigatória a atribuição de acento tonal a palavras prosódicas cabeça de frase fonológica (ver exemplos em (3a) e (3c)). Na mesma variedade de português: (i) em contexto de focalização, a fronteira direita da frase fonológica é relevante na atribuição de acento frasal (FERNANDES, 2007; TENANI e FERNANDES-SVARTMAN, 2008), ${ }^{14}$ como se observa em (4); e (ii) a frase entoacional (I) e o enunciado fonológico (U) são domínios relevantes para a atribuição de tons de fronteira (T\% no exemplo em (5)) (FROTA; VIGÁRIO, 2000; TENANI, 2002; SERRA, 2009), ${ }^{15}$ como se nota em (5).

(4) Contexto: Quem leu os livros?

[O João]PPh leu os livros. ${ }^{16}$

$\mathrm{T}^{\mathrm{T}}$

(5) [[O João]I [segundo dizem]I [é um excelente aluno]I/U

$$
\begin{array}{lll}
\mathrm{T} \% & \mathrm{~T} \% & \mathrm{~T} \%
\end{array}
$$

Uma vez já expostos os argumentos que justificam a adoção da visão integrada entre as teorias Fonologia Entoacional e Fonologia Prosódica, apresentaremos, brevemente, os pressupostos teóricos dessas duas teorias necessários para $\mathrm{o}$ desenvolvimento deste trabalho.

\subsection{Fonologia Eentoacional}

De acordo com a Fonologia Entoacional (PIERREHUMBERT, 1980; BECKMAN; PIERREHUMBERT, 1986; LADD, 1996, 2008; entre outros), a entoação tem uma organização fonológica e um dos principais objetivos dessa teoria é tentar fornecer um aparato descritivo universal para a entoação.

Nessa perspectiva, um contorno entoacional consiste, fonologicamente, em uma sequência de unidades discretas, os eventos tonais. Esses eventos são localmente definidos e associados a pontos específicos na cadeia segmental. A representação fonética de uma sequência de eventos tonais consiste no contorno da frequência fundamental $\left(\mathrm{F}_{0}\right)$.

Os eventos tonais são compostos por níveis de tons primitivos ou por alvos da altura, alto (H - high) e baixo (L - low), e são classificados como como acentos tonais ou tons relacionados a fronteiras.

Os acentos tonais são associados a sílabas proeminentes, podendo ser simples, monotonais $\left(\mathrm{L}^{*}\right.$ ou $\left.\mathrm{H}^{*}\right)$, ou complexos, bitonais $\left(\mathrm{H}^{*}+\mathrm{L}, \mathrm{H}+\mathrm{L}^{*}, \mathrm{~L}^{*}+\mathrm{H}\right.$ ou $\left.\mathrm{L}+\mathrm{H}^{*}\right)$. Já os tons relacionados a fronteiras são associados a fronteiras de domínios prosódicos, considerando a visão integrada entre entoação e organização

\footnotetext{
${ }^{14}$ Sobre acento frasal, conferir seção 2.1 .

${ }^{15}$ Baseando-nos em Nespor e Vogel $(1986,2007)$, definimos o enunciado fonológico (U phonological utterance) como o domínio prosódico que abrange frases entoacionais relacionadas semanticamente.

${ }^{16} \mathrm{O}$ elemento focalizado aparece em negrito e " $\mathrm{T}$ " representa acento frasal.
} 
dos domínios prosódicos, e consistem nos tons de fronteira (boundary tones: L\% ou $\mathrm{H} \%$ ) e nos acentos frasais (phrasal accents: $\mathrm{L}^{-}$ou $\left.\mathrm{H}^{-}\right) .{ }^{17}$ Os tons de fronteira se encontram associados a fronteiras de domínios prosódicos mais altos da hierarquia prosódica, como a frase entoacional (I) e o enunciado fonológico (U), e os acentos frasais são associados a fronteiras de domínios mais baixos que I e U, como a frase fonológica, por exemplo.

\subsection{Fonologia Prosódica}

A Fonologia Prosódica é uma teoria da estrutura fonológica e sua relação com a sintaxe (SELKIRK, 1984, 1986, 2000; NESPOR; VOGEL, 1986, 2007; HAYES, 1989; entre outros).

Segundo essa teoria, o fluxo da fala é organizado hierarquicamente dentro de domínios prosódicos e a evidência para esta hierarquia prosódica provém da operação de regras fonológicas, que se aplicam no interior e entre junturas de certos domínios e que são bloqueadas no interior e entre junturas de outros.

A estrutura prosódica é parcialmente determinada pela estrutura sintática. Em alguns casos, essas duas estruturas podem coincidir, e, em outros, divergir. Essa é a razão por que a sintaxe nem sempre faz as previsões corretas sobre a estrutura prosódica.

A relação entre a estrutura sintática e a prosódica é definida por um mapeamento sintático-fonológico que fornece uma representação prosódica consistindo em uma hierarquia de constituintes prosódicos.

Tal hierarquia, na perspectiva de Nespor e Vogel (1986), por exemplo, é disposta como indicado na Figura 1, incluindo desde a sílaba até o enunciado.

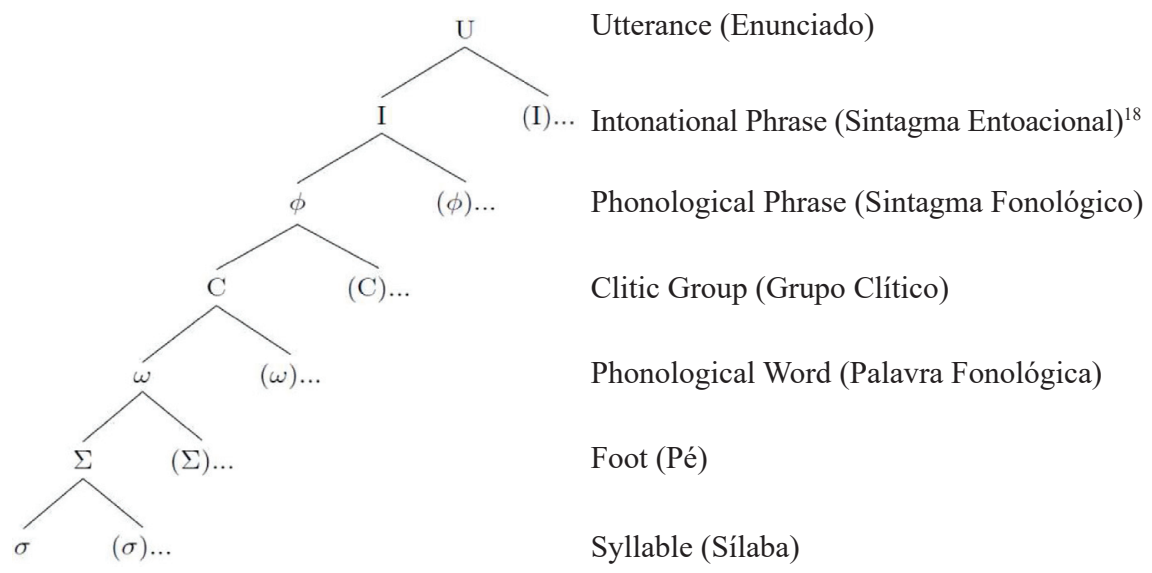

Figura 1: Representação da Hierarquia Prosódica segundo

Nespor e Vogel $(1986,2007)$ e adaptada de Bisol (2005, pp. 244).

${ }^{17} \mathrm{Na}$ literatura linguística brasileira sobre entoação do PB, "acento frasal" é o termo mais utilizado para a tradução de phrasal accent.

${ }_{18}$ Intonational phrase e phonological phrase são traduzidos, respectivamente, como "frase entoacional" ou "sintagma entoacional" e "frase fonológica" ou "sintagma fonológico" na literatura linguística brasileira sobre Fonologia Prosódica. 
Uma vez que a palavra fonológica, ou palavra prosódica (termo adotado neste trabalho) é o domínio relevante para a associação de acentos tonais em português brasileiro, como comprovado estatisticamente por Toneli (2014), será esse o constituinte prosódico privilegiado em nossa análise de dados. Conforme já mencionado anteriormente, a palavra prosódica é o domínio no qual pode haver apenas um acento primário (ou lexical).

\section{CORPUS E METODOLOGIA}

\subsection{Corpus}

O corpus utilizado em nossa análise refere-se a dados de fala provenientes da leitura da versão adaptada para o PB do Romance Languages Database RLD (D'IMPERIO et al., 2005; ELORDIETA et al., 2005; FROTA et al., 2011), corpus previamente construído para o estudo prosódico das línguas românicas e que é composto por 76 sentenças declarativas neutras na ordem "sujeito (S) - verbo (V) - objeto (O)", controladas em termos de complexidade sintática e prosódica (ramificação/ não ramificação de constituintes) e quanto à extensão dos constituintes em número de sílabas. Essas sentenças foram elaboradas com todas as combinações de condições de extensão de constituintes (duas condições: constituintes curtos, compostos por três sílabas, e constituintes longos, compostos por cinco ou mais sílabas) e de ramificação sintática e prosódica (três condições: constituintes não ramificados, formados por um núcleo lexical/uma palavra prosódica (PW), constituintes ramificados, formados por dois núcleos lexicais/ duas PWs, e constituintes duplamente ramificados, formados por mais de dois núcleos lexicais/mais de duas PWs). Exemplos de sentenças do corpus são apresentados em (6), (7), (8) e (9).

(6) Sujeito (S) curto não-ramificado, verbo (V) curto e objeto (O) curto não ramificado ( $\mathrm{S}=3$ sílabas; $\mathrm{V}=3$ sílabas; $\mathrm{e} \mathrm{O}=3$ sílabas):

A nora mimava a noiva.

(7) Sujeito longo não-ramificado, verbo longo e objeto longo não-ramificado ( $\mathrm{S}=6$ sílabas; $\mathrm{V}=5$ sílabas; $\mathrm{e} \mathrm{O}=6$ sílabas):

A boliviana memorizava uma melodia.

(8) Sujeito ramificado curto, verbo curto e objeto curto ( $\mathrm{S}$ composto por substantivo + adjetivo $=5$ sílabas; $\mathrm{V}=3$ sílabas; e $\mathrm{O}=3$ sílabas):

A jovem loira levava a linha.

(9) Sujeito longo ramificado, verbo curto e objeto curto não-ramificado ( $\mathrm{S}$ composto por substantivo + adjetivo $=10$ sílabas; $\mathrm{V}=3$ sílabas; $\mathrm{e} \mathrm{O}=3$ sílabas):

A libanesa maravilhosa levava a noiva. 
O conjunto de dados analisado aqui refere-se a arquivos sonoros provenientes da gravação digital da leitura, incluindo três repetições, das 76 sentenças já referidas realizada por duas falantes do dialeto paulista do $\mathrm{PB}$, de mesmo nível de escolaridade e mesma faixa etária. $\mathrm{O}$ total de sentenças que compõe esse conjunto de dados é 456 ( 76 sentenças x 2 falantes x 3 repetições).

\subsection{Metodologia}

A metodologia de obtenção dos dados consistiu na gravação digital da leitura das 76 sentenças referidas na subseção 3.1, ordenadas aleatoriamente e intercaladas com sentenças distratoras. A leitura das 76 sentenças incluiu três repetições e foi realizada por duas falantes (B e D) do dialeto paulista do PB (cidade de São Paulo), de nível escolar superior e de faixa etária entre 20 e 45 anos.

Por sua vez, a metodologia empregada na análise de dados consistiu: (i) na segmentação das sentenças do corpus em sílabas e palavras; (ii) na transcrição dos eventos tonais associados ao contorno entoacional dessas sentenças, com base na análise perceptual e acústica, levando em conta os pressupostos teóricos da Fonologia Entoacional (PIERREHUMBERT, 1980; BECKMAN; PIERREHUMBERT, 1986; LADD 1996, 2008; e, especificamente para o PB, FROTA; VIGÁRIO, 2000; TENANI, 2002; FERNANDES, 2007; TRUCKENBRODT et al., 2009; VIGÁRIO; FERNANDES-SVARTMAN, 2010; TONELI, 2014; FROTA et al., 2015; entre outros) e através do uso do programa computacional de análise de fala Praat (BOESRMA; WEENINK, 2010); (iii) na quantificação dos acentos tonais (T*) e de tons adicionais encontrados associados às PWs que compõem os sujeitos e predicados das sentenças; ${ }^{19}$ (iv) no cálculo da densidade tonal, definida aqui como proporção de T* por PW, nestes constituintes sintáticos; e (v) na aplicação de testes estatísticos para a confirmação ou infirmação da diferença ou igualdade entre os valores de densidade tonal encontrados para os já referidos constituintes sintáticos.

\section{DESCRIÇÃO E ANÁLISE DOS RESULTADOS}

Das 456 sentenças produzidas, foi analisado um total de 455, porque houve problemas técnicos na gravação de uma das sentenças produzidas pela falante B.

Os resultados alcançados confirmam a hipótese deste trabalho, segundo a qual, além de fatores como número de sílabas e ramificação sintática e prosódica, a posição sintática ocupada pelas PWs nas sentenças também desempenha um papel relevante na associação de acentos tonais ao contorno entoacional neutro do PB. Ainda que pareça, em um primeiro momento, ser quase categórica a associação de um acento tonal a cada PW que compõe as sentenças, uma observação mais atenta dos resultados obtidos da análise de dados aqui empreendida revela diferenças importantes entre a associação tonal às PWs que compõem o sujeito e a atribuição tonal às PWs que compõem o predicado.

Sobre tons adicionais em sentenças neutras do PB, conferir, entre outros, Frota e Vigário (2000), Tenani (2002), Fernandes-Svartman (2009) e Toneli (2014). Para Tenani (2002) e Fernandes-Svartman (2009), esse tipo de tom está relacionado à sílaba portadora de acento secundário em PB. 
Através da observação dos resultados apresentados no Quadro 1, nota-se a densidade tonal de $100 \%$ ou densidades muito próximas a esse valor ( $98 \%$ e $99 \%$ ) em todos os tipos de sujeito (S) das sentenças por nós analisadas: (i) densidade tonal de $100 \%$ no caso de sujeito curto não ramificado (formado por $1 \mathrm{PW}$ e até três sílabas, ex.: “a jovem”), de sujeito longo não ramificado (formado por 1 PW e 5 ou mais sílabas, ex.: "a libanesa") e de sujeito longo ramificado (formado por 2 PWs, sendo cada PW composta por 5 ou mais sílabas, ex.: "a libanesa maravilhosa”); (ii) densidade tonal de $98 \%$ no caso de sujeito curto ramificado (formado por 2 PWs, sendo cada PW composta por até três sílabas, ex.: "a jovem loira") e de sujeito longo duplamente ramificado (formado por mais de 2 PWs, sendo cada PW composta por 5 ou mais sílabas, ex.: "a libanesa maravilhosa de Borborema"); e (iii) densidade tonal de $99 \%$ no caso de sujeito curto duplamente ramificado (formado por mais de 2 PWs, sendo cada PW composta por até 3 sílabas, ex.: "a nora morena da velha"). Ou seja, há a atribuição praticamente categórica de um acento tonal para cada PW que compõe os sujeitos das sentenças do nosso corpus. Além dos acentos tonais, também se verificam tons adicionais associados a sílabas pretônicas de PWs longas que compõem os sujeitos das sentenças. Como pode ser observado na quarta coluna do Quadro 1, esse tipo de tom foi encontrado em 34 das 96 ocorrências de sujeito longo não ramificado, em 21 das 96 ocorrências de sujeito longo ramificado e em 1 das 35 ocorrências de sujeito longo duplamente ramificado.

Quadro 1: Densidade tonal, número de acentos tonais, PW e tons adicionais em PWs longas nos diferentes tipos de sujeito.

\begin{tabular}{|l|c|c|c|c|}
\hline \multicolumn{1}{|c|}{ Tipo de S } & No. de PWs & No. de T* & $\begin{array}{c}\text { Ts adicionais em } \\
\text { PWs longas }\end{array}$ & $\begin{array}{c}\text { Densidade } \\
\text { tonal }\end{array}$ \\
\hline $\begin{array}{l}\text { Scurto não-ramificado } \\
\text { (96 casos) }\end{array}$ & 96 & 96 & - & $100 \%$ \\
\hline $\begin{array}{l}\text { Slongo não-ramificado } \\
\text { (96 casos) }\end{array}$ & 96 & 96 & 34 & $100 \%$ \\
\hline $\begin{array}{l}\text { Scurto ramificado } \\
\text { (96 casos) }\end{array}$ & 192 & 188 & - & $98 \%$ \\
\hline $\begin{array}{l}\text { Slongo ramificado } \\
\text { (96 casos) }\end{array}$ & 192 & 192 & 21 & $100 \%$ \\
\hline $\begin{array}{l}\text { Scurto duplamente } \\
\text { ramificado (36 casos) }\end{array}$ & 108 & 107 & - & $99 \%$ \\
\hline $\begin{array}{l}\text { Slongo duplamente } \\
\text { ramificado (35 casos) }\end{array}$ & 105 & 103 & 1 & $98 \%$ \\
\hline
\end{tabular}

A representação em (10), bem como a respectiva Figura 2, ilustram um caso de associação de um acento tonal a cada PW que compõe um sujeito longo e ramificado (um acento tonal $\mathrm{L}^{*}+\mathrm{H}$ associado a $\mathrm{PW}$ "a libanesa" e um acento tonal $\mathrm{L}^{*}+\mathrm{H}$ associado a PW "maravilhosa") e de associação de tom adicional a uma sílaba pretônica da primeira PW longa que compõe esse sujeito (associação do tom adicional H à sílaba pretônica "li" da PW "a libanesa"). 


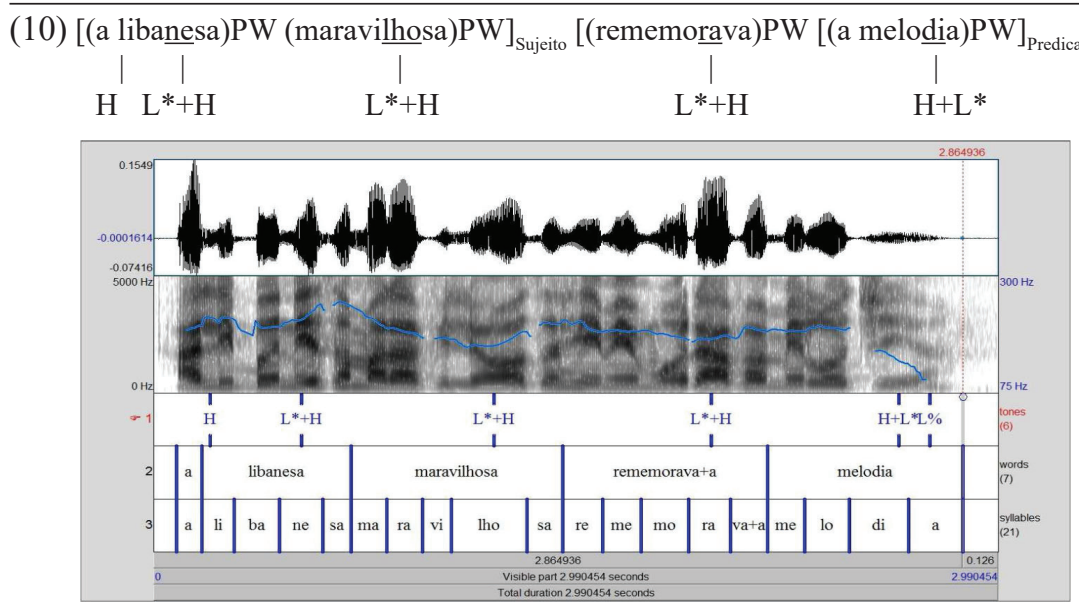

Figura 2: Segmentação e análise entoacional da sentença declarativa neutra "A libanesa maravilhosa rememorava a melodia", produzida pela falante D.

Quanto à densidade tonal nos predicados das sentenças analisadas, como pode ser observado no Quadro 2, embora a densidade tonal seja ainda alta para os diferentes tipos de predicado (de 82\% a 100\%), a associação tonal de um $\mathrm{T}^{*}$ a cada $\mathrm{PW}$ parece não ser categórica, uma vez que há valores de densidade tonal não tão próximos a $100 \%$, como é o caso de predicados formados por: (i) verbo curto e objeto curto ramificado (ex.: "levava liras na mala") - densidade tonal de $82 \%$; (ii) verbo longo e objeto curto ramificado (ex.: "manuseava liras na mala") - densidade tonal de $89 \%$; (iii) verbo curto e objeto curto duplamente ramificado (ex.: "levava a nora morena da velha") - densidade tonal de $91 \%$; e (iv) verbo curto e objeto longo duplamente ramificado (ex.: "gravava a serenata maravilhosa da Madalena") - densidade tonal de 94\%. Nesses tipos de predicados de nossas sentenças, é possível a atribuição de acento tonal apenas a $\mathrm{PWs}$ cabeça de $\mathrm{PPh}$. No que diz respeito à associação de tons adicionais, esse tipo de tom também é encontrado associado a sílabas pretônicas de PWs longas do predicado, entretanto, os tons adicionais são encontrados em menor número ao compararmos com a quantidade de tons adicionais associados a PWs longas que compõem os sujeitos das sentenças. Conforme os resultados apresentados na quarta coluna do Quadro 2, tons adicionais foram encontrados em 1 das 48 ocorrências de predicado formado por verbo curto e objeto longo ramificado, em 1 das 48 ocorrências de predicado formado por verbo longo e objeto longo ramificado, em 10 das 18 ocorrências de predicado formado por verbo curto e objeto longo duplamente ramificado e em 11 das 18 ocorrências de predicado formado por verbo longo e objeto longo duplamente ramificado. 
Quadro 2: Densidade tonal, número de acentos tonais, $\mathrm{PW}$ e tons adicionais em PWs longas nos diferentes tipos de predicado.

\begin{tabular}{|c|c|c|c|c|}
\hline Tipo de Predicado & No. de PWs & No. de $T^{*}$ & $\begin{array}{l}\text { Ts adicionais } \\
\text { em PWs longas }\end{array}$ & $\begin{array}{c}\text { Densidade } \\
\text { tonal }\end{array}$ \\
\hline $\begin{array}{l}\text { Vcurto + Ocurto } \\
\text { não-ramificado } \\
\text { ( } 48 \text { casos) }\end{array}$ & 96 & 94 & - & $98 \%$ \\
\hline $\begin{array}{l}\text { Vlongo + Ocurto } \\
\text { não-ramificado } \\
\text { ( } 48 \text { casos) }\end{array}$ & 96 & 95 & 0 & $99 \%$ \\
\hline $\begin{array}{l}\text { Vcurto + Olongo } \\
\text { não-ramificado } \\
\text { ( } 48 \text { casos) }\end{array}$ & 96 & 93 & 0 & $97 \%$ \\
\hline $\begin{array}{l}\text { Vlongo + Olongo } \\
\text { não-ramificado } \\
\text { (47 casos) }\end{array}$ & 94 & 94 & 0 & $100 \%$ \\
\hline $\begin{array}{l}\text { Vcurto + Ocurto } \\
\text { ramificado ( } 48 \text { casos) }\end{array}$ & 144 & 118 & - & $82 \%$ \\
\hline $\begin{array}{l}\text { Vlongo + Ocurto } \\
\text { ramificado ( } 48 \text { casos) }\end{array}$ & 144 & 128 & 0 & $89 \%$ \\
\hline $\begin{array}{l}\text { Vcurto + Olongo } \\
\text { ramificado ( } 48 \text { casos) }\end{array}$ & 144 & 143 & 1 & $99 \%$ \\
\hline $\begin{array}{l}\text { Vlongo + Olongo } \\
\text { ramificado ( } 48 \text { casos) }\end{array}$ & 144 & 142 & 1 & $98 \%$ \\
\hline $\begin{array}{l}\text { Vcurto + Ocurto } \\
\text { duplamente } \\
\text { ramificado ( } 17 \text { casos) }\end{array}$ & 68 & 62 & - & $91 \%$ \\
\hline $\begin{array}{l}\text { Vlongo + Ocurto } \\
\text { duplamente } \\
\text { ramificado (18 casos) }\end{array}$ & 72 & 69 & 0 & $96 \%$ \\
\hline $\begin{array}{l}\text { Vcurto + Olongo } \\
\text { duplamente } \\
\text { ramificado (18 casos) }\end{array}$ & 72 & 68 & 10 & $94 \%$ \\
\hline $\begin{array}{l}\text { Vlongo + Olongo } \\
\text { duplamente } \\
\text { ramificado (18 casos) }\end{array}$ & 72 & 69 & 11 & $96 \%$ \\
\hline
\end{tabular}

A representação em (11), bem como a respectiva Figura 3, consistem em um exemplo de caso de associação de acento tonal apenas à PW cabeça de PPh em predicado formado por verbo curto e objeto curto duplamente ramificado do nosso corpus. Conforme o exemplo, no primeiro PPh que compõe o objeto "a nora morena da velha" da sentença "O homem levava a nora morena da velha", há associação de acento tonal $\left(\mathrm{L}^{*}+\mathrm{H}\right)$ apenas à $\mathrm{PW}$ cabeça, (morena) PW, desse $\mathrm{PPh}$, nomeadamente, [(a nora)PW (morena)PW]PPh. 
(11)

[[(o homem)PWd]PPh] [(levava)PWd]PPh [(a nora)PWd (morena)PW]PPh[(da velha)PW]PPh]
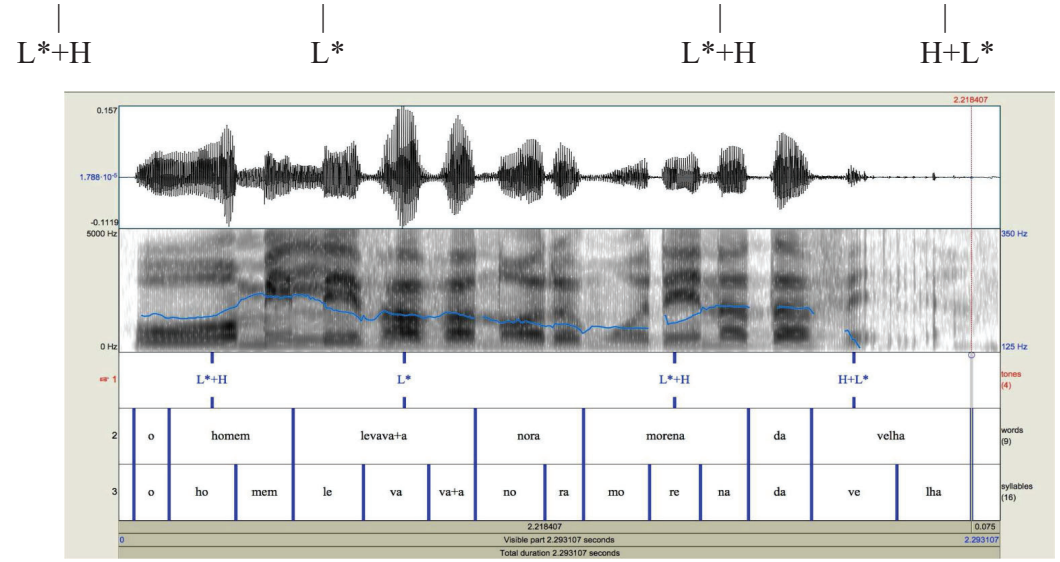

Figura 3: Segmentação e análise entoacional da sentença declarativa neutra "O homem levava a nora morena da velha", produzida pela falante D.

Dos resultados de densidade tonal nos sujeitos e predicados apresentados respectivamente no Quadro 1 e no Quadro 2, depreendemos as seguintes observações: (i) em sujeitos e objetos não ramificados, há a associação de um acento tonal a cada PW, independentemente do tamanho de PW em número de sílabas; (ii) há possibilidade de associação de tom adicional a sílabas pretônicas em PWs longas do sujeito e do predicado, sendo mais frequente esse tipo de associação tonal em PWs longas do sujeito; e (iii) em predicados formados por PPhs ramificados, é possível a associação de acento tonal apenas à $\mathrm{PW}$ cabeça de $\mathrm{PPh}$.

Por sua vez, da comparação entre os valores gerais de densidade tonal dos sujeitos e predicados apresentados nos já referidos Quadro 1 e Quadro 2, supõese que os sujeitos apresentam densidade tonal maior que os predicados, embora a densidade tonal nesses dois tipos de consituitintes seja alta nas sentenças (acima de 82\%).

Essa suposição se confirma ao aplicarmos testes estatísticos aos resultados obtidos. ${ }^{20}$ Primeiramente, calculamos a média e a variância das densidades tonais no sujeito e no predicado das sentenças. Os resultados obtidos a partir desses cálculos são apresentados no Quadro 3.

Quadro 3: Valores obtidos do cálculo da média e da variância da densidade tonal dos constituintes sujeito e predicado das sentenças do corpus.

\begin{tabular}{|c|c|c|}
\hline Constituinte & Média & Variância \\
\hline Sujeito & $99,167 \%$ & 0,967 \\
\hline Predicado & $94,917 \%$ & 27,538 \\
\hline
\end{tabular}

Agradecemos a Profa. Verónica Andrea González-López pelas críticas feitas à análise estatística realizada neste trabalho. 
Para averiguarmos se a diferença entre as médias das densidades tonais dos constituintes sujeito e predicado é estatisticamente significativa, aplicamos dois testes estatísticos não paramétricos. Isso porque os valores de variância dos dois grupos, "sujeito" e "predicado", são muito diferentes (0,967 para sujeito vs. 27,538 para predicado), comprometendo a validade da aplicação de um teste estatístico paramétrico mais usual como, por exemplo, o teste t-student. Os testes não paramétricos utilizados foram testes de permutação utilizando o p-valor exato ou com aleatorização. Esses testes foram realizados por meio da ferramenta computacional de análise estatística $\mathrm{R}$ (versão 3.3.3), através do uso das funções “perm.test”, que utiliza o p-valor exato, do pacote "jmOutlier" (https://cran.rproject.org/web/packages/jmuOutlier/jmuOutlier. pdf) e "perm.t.test", que utiliza aleatorização, do pacote "Deducer" (https://cran. rproject.org/web/packages/Deducer/Deducer.pdf) da referida ferramenta.

Para os dois testes, a hipótese nula considerada foi a de que a densidade tonal é igual nos dois grupos, "sujeito" (S) e "predicado" (Pred), e as hipóteses alternativas foram as seguintes: (i) a densidade tonal é diferente entre os grupos "sujeito" e "predicado"; e (ii) a densidade tonal do grupo "sujeito" é, em média, maior que a densidade tonal do grupo "predicado".

No Quadro 4, encontram-se os p-valores obtidos da aplicação de cada teste:

Quadro 4: P-valores obtidos da aplicação dos testes de permutação utilizando o p-valor exato e de permutação com aleatorização"

\begin{tabular}{|c|c|c|}
\hline Testes & $\begin{array}{c}\text { Hipótese alternativa (i): } \\
\text { densidade tonal de S difere } \\
\text { da densidade tonal de Pred }\end{array}$ & $\begin{array}{c}\text { Hipótese alternativa (ii): } \\
\text { densidade tonal de } \mathbf{S}>\text { densidade } \\
\text { tonal de Pred }\end{array}$ \\
\hline $\begin{array}{c}\text { Permutação com } \\
\text { p-valor exato } \\
\text { (perm.test) }\end{array}$ & 0,06092 & 0,01045 \\
\hline $\begin{array}{c}\text { Permutação com } \\
\text { aleatorização } \\
\text { (perm.t.test) }\end{array}$ & 0,01446 & 0,00737 \\
\hline
\end{tabular}

Considerando p-valor $=0,05$ e de acordo com os resultados apresentados no Quadro 4, rejeita-se a hipótese nula de igualdade entre as densidades tonais dos constituintes sujeito e predicado em nossos dados, uma vez que, da aplicação do teste de permutação com aleatorização, foi obtido p-valor $=0,01446(<0,05)$ em favor da hipótese alternativa de que a densidade tonal é diferente entre esses constituintes (hipótese alternativa (i)).

Por sua vez, os p-valores de 0,01045 $(<0,05)$ e $0,00737(<0,05)$, apresentados no mesmo quadro e obtidos da aplicação dos testes de permutação com p-valor exato e de permutação com aleatorização, respectivamente, confirmam que a densidade tonal nos sujeitos é, em média, maior que a densidade tonal nos predicados das sentenças (hipótese alternativa (ii)). 


\section{CONSIDERAÇÕES FINAIS}

Neste trabalho, baseando-nos na análise de dados da variedade paulista do $\mathrm{PB}$, visamos ao estudo dos fatores determinantes na associação de acentos tonais ao contorno de sentenças declarativas neutras dessa variedade de português. Nossa hipótese era a de que, além de fatores como número de sílabas e ramificação sintática e prosódica, a posição sintática ocupada pelas PWs nas sentenças também poderia desempenhar um papel relevante na associação de acentos tonais ao contorno entoacional neutro do PB.

Os resultados alcançados de nossa análise de dados confirmaram essa hipótese, na medida em que foram encontradas diferenças entre a associação de acentos tonais às PWs que compõem o sujeito e a associação de acentos tonais às PWs que compõem o predicado: nos sujeitos, a associação tonal de um acento tonal a cada PW é praticamente categórica e, nos predicados das sentenças, é possível a associação de acento tonal apenas à $\mathrm{PW}$ cabeça de $\mathrm{PPh}$. Essa diferença resulta em uma densidade tonal média maior, comprovada estatisticamente (ver seção 4), no sujeito do que nos predicados das sentenças de nosso corpus. Ainda quanto à diferença entre a associação tonal no sujeito e no predicado, também foi notado que PWs longas apresentam mais frequentemente um tom adicional associado a uma sílaba pretônica quando compõem o sujeito do que quando compõem o predicado das sentenças.

Tais resultados indicam que os sujeitos dos nossos dados da variedade paulista do PB são marcados prosodicamente de maneira mais proeminente do que os predicados, no que se refere a uma maior atribuição de tons (acentos tonais e tons adicionais). É possível que essa maior proeminência prosódica do sujeito reflita propriedades sintáticas especiais desse constituinte em $\mathrm{PB}$, como o fato de o sujeito consistir em um sujeito-tópico, considerando que essa variedade de português é orientada para o tópico, como afirmam Pontes (1983), Galves (1998, 2001), entre outros.

Levando em conta tais resultados, surgem as seguintes indagações a serem exploradas em trabalhos futuros: 1. A atribuição tonal diferenciada para os elementos componentes do sujeito em PB poderia sinalizar particularidades sintáticas desse constituinte (como posição sintática) à semelhança do que afirmam Elordieta et al. (2005) e Frota e Vigário (2007) para o fraseamento entoacional de outras línguas/variedades românicas como o espanhol, o português europeu padrão e o português europeu do Norte? 2. A associação de acentos tonais em PB respeita ou não critérios de uniformidade de fraseamento no sujeito e no predicado, como afirmam Sandalo e Truckenbrodt (2002) com base na análise de dados de retração de acento dessa mesma variedade?

Para a investigação aprofundada dessas questões, dados de mais falantes e de outras variedades do PB, além de dados de fala espontânea, devem ser incluídos nas análises a serem desenvolvidas em trabalhos futuros. 


\section{REFERÊNCIAS BIBLIOGRÁFICAS}

ABAURRE, M. B. M. Acento frasal e processos fonológicos segmentais. Letras de Hoje, v. 31, n. 2, pp. 41-50, 1996.

ABOUSAlH, E. Resolução de Choques de Acento No Português Brasileiro: Elementos Para Uma Reflexão Sobre A Interface Sintaxe-Fonologia. 1997. Dissertação (Mestrado em Linguística) Universidade Estadual de Campinas, Campinas, 1997.

BECKMAN, M.; PIERREHUMBERT, J. Intonational structure in Japanese and English. Phonology Yearbook, v. 3, n. 1, pp. 255-309, 1986.

BISOL, B. O sândi e a ressilabação. Letras de Hoje, v.31, n.2, pp. 159-168, 1996.

BISOL, L. Os constituintes prosódicos. In: BISOL, L. (Org.) Introdução a estudos de fonologia do português brasileiro, 4a. edição revista e ampliada. Porto Alegre: EDIPUCRS, 2005, pp. 243-255.

BOERSMA, P.; WEENINK, D. Praat: doing phonetics by computer [Computer Program]. Versão 5.2.07. Disponível em: <http//: www.praat.org/>. Acesso em: 24 dez. 2010.

D'IMPERIO, M.; ELORDIETA, G.; FROTA, S.; PRIETO, P.; VIGÁRIO, M. Intonational phrasing in Romance: The role of syntactic and prosodic structure. In: FROTA, S; VIGÁRIO, M.; FREITAS, M. J. (Eds.) Prosodies. Berlin/New York: Mouton de Gruyter, 2005, pp. 59-97.

ELORDIETA, G.; FROTA, S.; VIGÁRIO, M. Subjects, objects and intonational phrasing in Spanish and Portuguese. Studia Linguistica, v. 59, n. 2-3, pp. 110-143, 2005.

FERNANDES, F. R. Ordem, focalização e preenchimento em português: sintaxe e prosódia. 2007. Tese (Doutorado em Lingüística) - Universidade Estadual de Campinas, Campinas, 2007.

FERNANDES-SVARTMAN, F. R. Acento secundário, atribuição tonal e ênfase em português brasileiro (PB). Estudos Lingüísticos, São Paulo, v. 38, n. 1, pp. 47-58, 2009.

FROTA, S. Prosody and focus in European Portuguese: Phonological phrasing and intonation. New York: Garland Publishing, 2000.

FROTA, S.; CRUZ, M.; FERNANDES-SVARTMAN, F.; COLLISCHONN, G.; FONSECA, A.; SERRA, C.; OLIVEIRA, P.; VIGÁRIO, M. Intonational variation in Portuguese: European and Brazilian varieties. In: FROTA, S.; PRIETO, P. (Eds.), Intonation in Romance. Oxford: Oxford University Press, 2015, pp. 235-283.

FROTA, S.; CRUZ, M.; VIGÁRIO, M. RLD - Romance Languages Database: Inline database for intonational phrasing in Romance. Version 1.0. Laboratório de Fonética (CLUL), Faculdade de Letras da Universidade de Lisboa, 2011. Disponível em: <http://rld.letras.ulisboa.pt/>.

FROTA, S.; VIGÁRIO, M. Aspectos de prosódia comparada: ritmo e entoação no PE e no PB. In: CAStro, R. V.; BARBOSA, P. (Orgs.) Actas do XV Encontro Nacional da Associação Portuguesa de Linguística. Coimbra: APL, 2000, v.1, pp. 533-555.

FROTA, S.; VIGÁRIO, M. Intonational phrasing in two varieties of European Portuguese. In: RIAD, T.; GUSSENHOVEn, C. (Eds.) Tones and Tunes, v. 1. Berlin: Mouton de Gruyter, 2007, pp. 265-291.

GALVES, C. M. C. Tópicos e sujeitos, pronomes e concordância no Português do Brasil. Cadernos de Estudos Linguísticos, v. 34, pp. 19-32, 1998. 
GALVES, C. M. C. Ensaios sobre as gramáticas do português. Campinas: Editora da Unicamp, 2001.

HAYES, B. The prosodic hierarchy in meter. In: KIPARSKY, P.; YOUMANS, G. Phonetics and Phonology - Rhythm and Meter, v. 1. San Diego, California: Academic Press, INC.,1989, pp. 201-260.

HAYES, B.; LAHIRI, A. Bengali intonational phonology. Natural Language \& Linguistic Theory, v. 9, n. 1, pp. 47-96, 1991.

JUN, S-A. The phonetics and phonology of Korean prosody: intonational phonology and prosodic structure. New York: Garland Publishing, 1996.

LADD, D. R. Intonational Phonology. Cambridge: Cambridge University Press,1996.

LADD, D. R. Intonational Phonology (2nd ed.). Cambridge: Cambridge University Press, 2008.

NESPOR, M.; VOGEL, I. Prosodic Phonology. Dordrecht: Foris Publications, 1986.

NESPOR, M.; VOGEL, I. Prosodic phonology: With a new foreword. Berlin/New York: Mouton de Gruyter, 2007.

PIERREHUMBERT, J. The phonology and phonetics of English intonation. 1980. Tese (Doutorado) - M.I.T., Cambridge, Mass., 1980.

PONTES, E. O tópico no português do Brasil. Campinas: Pontes, 1987.

SANDALO, F.; TRUCKENBRODT, H. Some notes on Phonological Phrasing in Brazilian Portuguese. Mit Working Papers In Linguistics, v. 42, pp. 285-310, 2002.

SELKIRK, E. O. Phonology and Syntax: The Relation between Sound and Structure. Cambridge: The M.I.T. Press, 1984.

SELKIRK, E. O. On derived domains in sentence phonology. Phonology Yearbook, n. 3, pp. 371-405, 1986.

SELKIRK, E. O. The interaction of constraints on prosodic phrasing. In: HORNE, M. (Ed.). Prosody: Theory and Experiment. Netherlands: Kluwer Academic Publishers, 2000, pp. 231-261.

SCHWINDT, L. C. O prefixo do português brasileiro: análise morfofonológica. 2000. Tese (Doutorado) - PUCRS, Porto Alegre, 2000.

SERRA, C. R. Realização e percepção de fronteiras prosódicas no português do Brasil: fala espontânea e leitura. 2009. Tese (Doutorado) - Universidade Federal do Rio de Janeiro, Rio de Janeiro, 2009.

SIMIONI, T. O clítico e seu lugar na estrutura prosódica do Português Brasileiro. ALFA, v. 52, n. 2, pp. 431-446, 2008.

TENANI, L. E. Domínios prosódicos no português: Implicações para a prosódia e para a aplicação de processos fonológicos. 2002. Tese (Doutorado em Linguística) - Universidade Estadual de Campinas, Campinas, 2002.

TENANI, L. E; FERNANDES-SVARTMAN, F. R. Prosodic phrasing and intonation in neutral and subject-narrow-focus sentences of Brazilian Portuguese. In: Fourth Conference on Speech Prosody 2008, 2008, Campinas. Proceedings of Fourth Conference on Speech Prosody 2008. Campinas: RG/CNPq, 2008, pp. 445-448. 
TONELI, P. A palavra prosódica em português brasileiro. 2014. Tese (Doutorado em Linguística) Universidade Estadual de Campinas, Campinas, 2014.

TRUCKENBRODT, H.; SANDALO, M. F.; ABAURRE; M. B. M. Elements of Brazilian Portuguese intonation. Journal of Portuguese Linguistics, v. 8, pp. 75-114, 2009.

VIGÁRIO, M. The Prosodic Word in European Portuguese. Berlin: Mouton de Gruyter, 2003.

VIGÁRIO, M. O lugar do Grupo Clítico e da Palavra Prosódica Composta na hierarquia prosódica: uma nova proposta. In: LOBO, M.; COUTINHO, M. A. (Orgs.) XXII Encontro da Associação Portuguesa de Lingüística - Textos Seleccionados. Lisboa: Colibri Artes Gráficas, 2007, pp. 673-688.

VIGÁRIO, M. Prosodic structure between the Prosodic Word and the Phonological Phrase: Recursive nodes or an independent domain? The Linguistic Review, v. 27, n. 4, pp. 485-530, 2010.

VIGÁRIO, M.; FERNANDES-SVARTMAN, F. R. A atribuição de acentos tonais em compostos no português do Brasil. In: BRITO, A. M.; SILVA, F.; VELOSO, J.; FIÉIS, A. (Orgs.) $X X V$ Encontro da Associação Portuguesa de Lingüística - Textos Seleccionados. Porto: Tip. Nunes, Ltda - Maia, 2010, v. 1, pp. 769-786. 